\title{
Improved Asymptotic Capacity Lower Bound for OFDM System with Compressed Sensing Channel Estimation for Bernoulli Gaussian Channel
}

\author{
Slavche PEJOSKI, Venceslav KAFEDZISKI \\ Faculty of Electrical Engineering and Information Technologies, University Ss. Cyril and Methodius, Skopje, \\ Republic of Macedonia \\ slavchep@feit.ukim.edu.mk, kafedzi@feit.ukim.edu.mk \\ Manuscript received January 25, 2016
}

\begin{abstract}
We propose an improved capacity lower bound for OFDM system with compressed sensing channel estimation for Bernoulli-Gaussian channel. We improve the known capacity lower bound which is based on Lasso compressed sensing channel estimation, by replacing the Lasso based estimate with an MMSE estimate, known to be optimal in the MMSE sense and achievable with practical algorithms for a broad range of system parameters setup. Additionally, for the system with equi-powered pilot subcarriers we optimize the capacity lower bound by finding the optimal average fraction of pilot subcarriers used for channel estimation and optimal pilot to data power ratio given the average symbol power per subcarrier, and propose an optimization procedure with polynomial complexity.
\end{abstract}

\section{Keywords}

Pilot aided channel estimation, OFDM, Lasso compressed sensing, turbo compressed sensing, replica method, capacity lower bound

\section{Introduction}

The pilot aided channel estimation in OFDM over block fading channel with rich scattering, producing nonzero values for most of the channel taps, is a well elaborated topic [1], [2]. The increase of the utilized bandwidth in practical systems and the introduction of compressed sensing (CS) brought a significant interest for pilot aided sparse channel estimation in OFDM and, thus, many new estimation algorithms with suitable pilot allocation schemes were proposed [3-12]. Those works confirm the gain from using CS but they don't consider the capacity increase or the optimal parameter choice in the system. These issues for the case of Lasso based channel estimation in the asymptotic scenario were addressed in a recent paper [13]. In [13], the authors made a formal connection between the pilot aided OFDM channel estimation problem for a Bernoulli-Gaussian channel and the compressed sensing problem with partial DFT sensing ma- trix for a Bernoulli-Gaussian unknown vector [14]. To avoid correlation between the channel estimate and the estimation error, in [13] a new hybrid Lasso-MMSE estimator was proposed. For this estimator the MMSE was found using known results from the Replica method [14], [15] and the asymptotic capacity lower bound was derived based on [1], [16]. Additionally, using the asymptotic capacity lower bound, the authors in [13] proposed an optimization problem for finding the optimal average fraction of pilot subcarriers used for channel estimation and optimal pilot to data power ratio given the average symbol power per subcarrier, which was solved using grid based exhaustive search. Here we improve the asymptotic capacity lower bound from [13] by replacing the Lasso based Lasso-MMSE by pure MMSE estimator. The MMSE estimation error of the MMSE estimator can also be predicted using the Replica method [14] and can be achieved for broad range of system parameters using the Turbo compressed sensing recovery algorithm [17], [18]. The improved asymptotic capacity lower bound sheds light on the expected improvement from the utilization of optimal reconstruction algorithms in compressed sensing compared to the widely used Lasso based solutions. Additionally, we analyze the problem of finding an optimal average fraction of pilot subcarriers used for channel estimation and optimal pilot to data power ratio given the average symbol power per subcarrier and the applicability of convex optimization procedures to solve the problem in polynomial time.

The novelty of this paper comes from improving the asymptotic capacity lower bound for pilot aided OFDM system achieved by replacing the Lasso-MMSE with the MMSE channel estimate. Additional novelty comes from analyzing the problem of finding the optimal average fraction of pilot subcarriers used for channel estimation and the optimal pilot to data power ratio given the average symbol power per subcarrier in the sense of the applicability of the common polynomial time optimization procedures.

The paper is organized as follows. In Sec. 2 we derive the improved lower bound for the asymptotic capacity of the system and formulate the problem of finding an optimal 
average fraction of pilot subcarriers used for channel estimation and optimal pilot to data power ratio given the average symbol power per subcarrier. In Sec. 3 we give numerical results to illustrate the strengths of the improved asymptotic capacity lower bound and analyze the applicability of different algorithms for solving the optimization problem. Section 4 concludes the paper.

\section{System Description}

We analyze the same OFDM system as in [13] that has $N$ subcarriers. At the transmitter the $N \times 1$ vector $\mathbf{X}$, containing the data and the pilot symbols, experiences IDFT and is then preceded by a cyclic prefix of length $L$ ( $N$ is an integer multiple of $L$ ). Afterwards, the signal is sent through a channel with a discrete channel impulse response represented by an $N \times 1$ vector $\mathbf{h}$ that has maximum $L$ nonzero values at the beginning of the vector followed by $N-L$ zero values. We use a channel model where the first $L$ taps of $\mathbf{h}$ follow the Bernoulli-Gaussian model, i.e. each of the first $L$ taps may assume a nonzero value with probability $q$, and, when it does, its value is obtained as a zero mean circularly symmetric complex Gaussian random variable with variance $\frac{1}{L q}$, leading to $\mathbb{E}\left[\mathbf{h}^{H} \mathbf{h}\right]=1$. This way, the average number of nonzero channel taps is equal to $q L \ll L$. The term asymptotic behaviour, in the paper, refers to the scenario where $N \rightarrow \infty$ and $L \rightarrow \infty$, but the ratio $v=L / N$ remains constant such that $0<v \leq 1$. At the receiver, the cyclic prefix is removed and then the noisy signal $\mathbf{y}$ experiences a DFT to obtain an $N \times 1$ vector $\mathbf{Y}$ :

$$
\mathbf{Y}=\mathbf{X}_{c} \mathbf{H}+\mathbf{N}=\mathbf{X}_{c} \mathbf{F h}+\mathbf{N}
$$

where $\mathbf{F}$ is a non-unitary $N \times N$ DFT matrix and $\mathbf{X}_{c}$ is a diagonal matrix with the elements of $\mathbf{X}$ on the main diagonal, $\mathbf{N}$ is a zero mean circularly symmetric complex Gaussian random noise vector with covariance matrix $\sigma_{N}^{2} \mathbf{I}$, and $\mathbf{H}=\mathbf{F h}$ with $\mathbb{E}\left[\left|H_{i}\right|^{2}\right]=1$ for $i=1, \ldots, N$ where $\mathbf{H}=\left[H_{1}, \ldots, H_{N}\right]^{T}$ ( $T$ denotes transpose). We assume a block fading channel model where $B$ OFDM symbols experience the same channel, but the nonzero channel tap positions and the values of the channel coefficients are generated independently for each block and only the first OFDM symbol in each block is used in the channel estimation procedure.

The OFDM symbol used for channel estimation has $M$ pilot subcarriers $(\mathbb{E}[M]=p L)$ placed at positions $m_{1}, \ldots, m_{M}$ (integers such that $\left.1 \leq m_{1}<\ldots<m_{M} \leq N\right)$ ). In [13], it is shown that when the pilot positions are restricted to a subset of $\mathcal{L}=\left\{\frac{N}{L}(l-1)+1: l=1,2, \ldots, L\right\}$ and their positions $m_{1}, \ldots, m_{M}$ are chosen from the set $\mathcal{L}$ according to a Bernoulli model with probability $p$ then for equi-power pilots with powers $P_{X_{c p}}$, using several straightforward mathematical manipulations [13], we obtain:

$$
\mathbf{G}_{L}=\mathbf{A} \mathbf{F}_{L} \mathbf{h}_{L 1}+\mathbf{Z}_{L}
$$

where $\mathbf{F}_{L}$ is a unitary $L \times L$ DFT matrix. In (2) $\mathbf{h}_{L 1}$ is an $L \times 1$ vector generated according to the Bernoulli-Gaussian model with probability $q$ and variance $\gamma_{x}=\frac{P_{X_{c p}}}{q \sigma_{N}^{2}}, \mathbf{G}_{L}$ is an $L \times 1$ vector, $\mathbf{Z}_{L}$ is an $L \times 1$ zero mean circularly symmetric complex Gaussian random noise vector with covariance matrix $\mathbf{I}$ and $\mathbf{A}$ is an $L \times L$ diagonal matrix with nonzero entries on the main diagonal equal to one at positions $\left(m_{1}-1\right) \frac{L}{N}+1, \ldots,\left(m_{M}-1\right) \frac{L}{N}+1$. For the model in (2) the asymptotic behaviour $(L \rightarrow \infty)$ for different estimators can be predicted using the Replica method [14]. In [13] the authors predicted the MMSE error estimate of a reconstruction algorithm obtained by augmenting the Lasso estimator with an MMSE estimator. As explained in [14] this is not the optimal estimator in the MMSE sense. Namely, the lowest MMSE is obtained if using $\hat{\mathbf{h}}_{L 1}^{\mathrm{MMSE}}$, the MMSE estimate of $\mathbf{h}_{L 1}$ from (2), and that MMSE estimation error value can be found by solving the following system of equations (equation (346) inserted in (17b) both from [14] and equation (17a) using (37) both from [14]):

$$
\begin{aligned}
\delta_{2} & =\mathbb{E}\left[\left|h_{L 1 i}-\hat{h}_{L 1 i}^{\mathrm{MMSE}}\right|^{2}\right] \\
& =q \gamma_{x}-\frac{q}{\eta_{2}\left(1+\gamma_{x} \eta_{2}\right)} \int_{0}^{\infty} \frac{t e^{-\frac{t}{\gamma_{x} \eta_{2}}}}{1+\left(1+\gamma_{x} \eta_{2}\right) \frac{(1-q)}{q} \mathrm{e}^{-t}} \mathrm{~d} t \\
\eta_{2} & =\frac{\delta_{2}+1-\sqrt{\left(\delta_{2}+1\right)^{2}-4 p \delta_{2}}}{2 \delta_{2}} .
\end{aligned}
$$

A solution to (3) that refers to maximum $\eta_{2}$, which is of interest here, can be obtained by iteratively solving both equations in (3) using a starting point with $\eta_{2_{\text {start }}} \leq 1$. Even though the values in (3) are obtained using the Replica method, for a broad range of system parameters (as long as the ratio $\frac{p}{q}$ is not too low, the case of interest here), as presented in [17], [18] there exists an algorithm with polynomial complexity whose performance closely agrees with the theoretically predicted values, the Turbo compressed sensing reconstruction algorithm.

When $\delta_{2}$, the value of the MMSE estimation error, is available, using the derivation procedure from [13] the asymptotic capacity for an OFDM system with channel state information to receiver only, is bounded as:

$$
C \geq\left(1-\frac{p v}{B}\right) \mathbb{E}\left[\log _{2}\left(1+\frac{\left|\hat{H}_{i}\right|^{2} \gamma_{0}}{\left(1+\frac{p v}{B}\left(S_{p}-1\right)\right)\left(1+\frac{\delta_{2}}{S_{p}}\right)}\right)\right]
$$

where $S_{p}=P_{X_{c p}} / P_{X_{c d}}$ is the pilot to data symbol power ratio, $\hat{H}_{i}$ is the estimate of $H_{i}$ and $\gamma_{0}=\frac{\frac{p v}{B} P_{X_{c p}}+\left(1-\frac{p v}{B}\right) P_{X_{c d}}}{\sigma_{N}^{2}}$ is the average signal to noise ratio. Assuming exponential distribution of $\left|\hat{H}_{i}\right|^{2}$, the asymptotic capacity is bounded as:

$$
C \geq\left(1-\frac{p v}{B}\right) e^{-\frac{1}{P_{1}}} \operatorname{expint}\left(\frac{1}{P_{1}}\right) \log _{2} \mathrm{e}=\underline{C}
$$

where $P_{1}=\frac{\gamma_{0}\left(1-\delta_{2} \frac{1+\frac{p v}{B}\left(S_{p}-1\right)}{\gamma_{0} S_{p}}\right)}{\left(1+\frac{p v}{B}\left(S_{p}-1\right)\right)\left(1+\frac{\delta_{2}}{S_{p}}\right)}$ and expint $(x)=\int_{x}^{\infty} \frac{\mathrm{e}^{-t}}{t} d t$.

For fixed $q$ and $\gamma_{0}$, which are considered as system parameters, the optimal values of $S_{p}$ and $p$ can be found by maximizing the right-hand side of (5): 


$$
\begin{array}{ll}
\max _{p, S_{p}} & \underline{C} \\
\text { s.t. } & q \gamma_{x}-\frac{q}{\eta_{2}\left(1+\gamma_{x} \eta_{2}\right)} \int_{0}^{\infty} \frac{t \mathrm{e}^{-\frac{t}{\gamma x \eta_{2}}}}{1+\left(1+\gamma_{x} \eta_{2}\right) \frac{(1-q)}{q} \mathrm{e}^{-t}} \mathrm{~d} t \\
& =\frac{\eta_{2}-p}{\eta_{2}^{2}-\eta_{2}}
\end{array}
$$

where the constraint is associated with (3) and is obtained by solving for $\delta_{2}$ in the second equation of (3).

\section{Numerical Results}

The numerical results are obtained using Matlab. In the numerical results we compare the capacity lower bound obtained using the MMSE estimation with the capacity lower bound obtained when using the Lasso-MMSE estimator [13] and capacity lower bound for the MMSE estimation with $L$ pilot symbols $(p=1)$, which has a closed form solution and the lowest complexity of all three considered estimators. To obtain the results we used a grid based search where the search of $p$ was in the interval [1.5q 1] with a step of 0.025 , and the $S_{p}$ was in the interval [0.4 13] with a step of 0.2 . If not stated differently, we set $B=2$.

In Fig. 1 we show the improvement in the optimized capacity lower bound due to the used estimators for the case when $q=0.1$. The figure shows that the increase in capacity lower bound is significant for high $\gamma_{0}$ and this increase can be observed for both Lasso-MMSE over the MMSE with $p=1$ and MMSE with $p<1$ over Lasso-MMSE. This shows that the performance of a Lasso based compressed sensing estimation can be significantly improved.

To better understand the system performance shown in Fig. 1, in Fig. 2 we show the obtained optimal values of $p$ and $S_{p}$ as a function of $\gamma_{0}$ for the three approaches.

Figure 2 shows that in both systems, that use LassoMMSE and MMSE with $p<1$, the increased $\gamma_{0}$ leads to reduced number of used pilots (decreased $p$ ), and increased power of the pilot symbols (increased $S_{p}$ ). Although the general trends are the same, the MMSE based system shows more pronounced behaviour. For the MMSE estimator with $p=1$ the value of $S_{p}$ decreases with the increase in $\gamma_{0}$.

In Fig. 3 we show the performance of the systems for variable $q$ when $\gamma_{0}=250$. Figure 3 shows that the increase in $q$ decreases the system optimized capacity lower bounds for all estimators. The improvement for the system using MMSE estimator over the system with Lasso-MMSE estimator is almost constant in the observed region. Interestingly, the system using Lasso-MMSE estimator shows improved performance over the MMSE with $p=1$ only for low values of $q$ and with the increase in $q$, the performance of both systems converge. This restricts the area where the utilization of Lasso based estimation can be implemented with reasonable gain over the system with $p=1$.

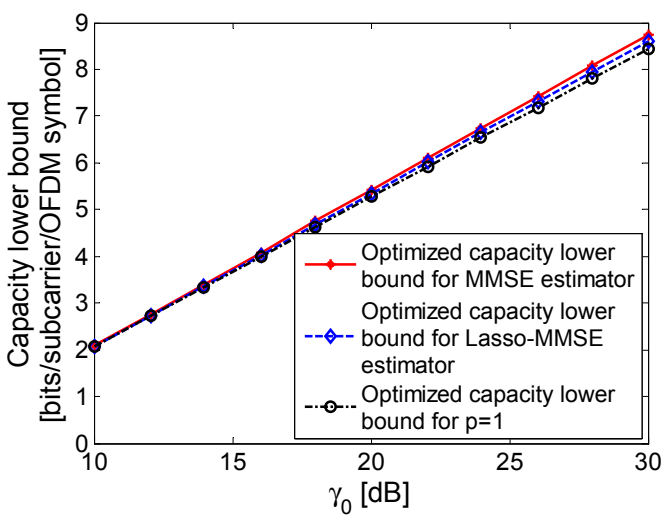

Fig. 1. Optimized capacity lower bound as a function of the average signal to noise ratio $\gamma_{0}$

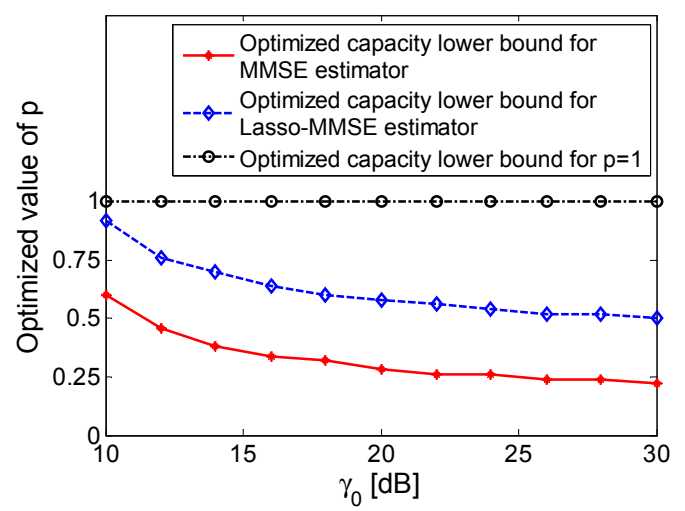

(a) Optimal $p$ as a function of $\gamma_{0}$

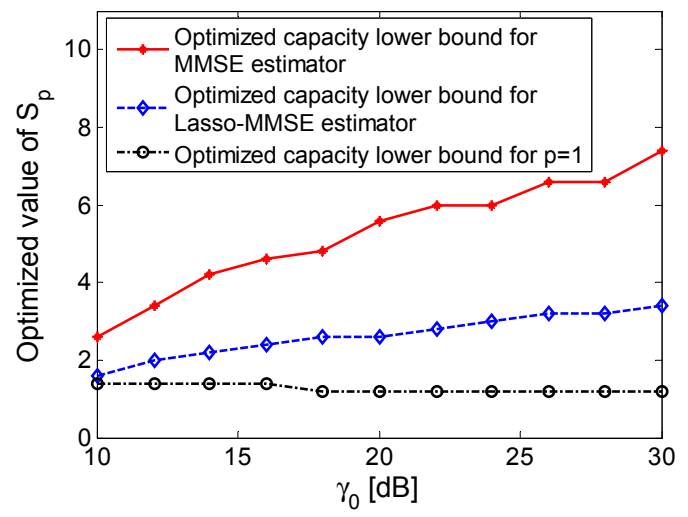

(b) Optimal $S_{p}$ as a function of $\gamma_{0}$

Fig. 2. Optimal $p$ and $S_{p}$ vs $\gamma_{0}$ for $q=0.1$.

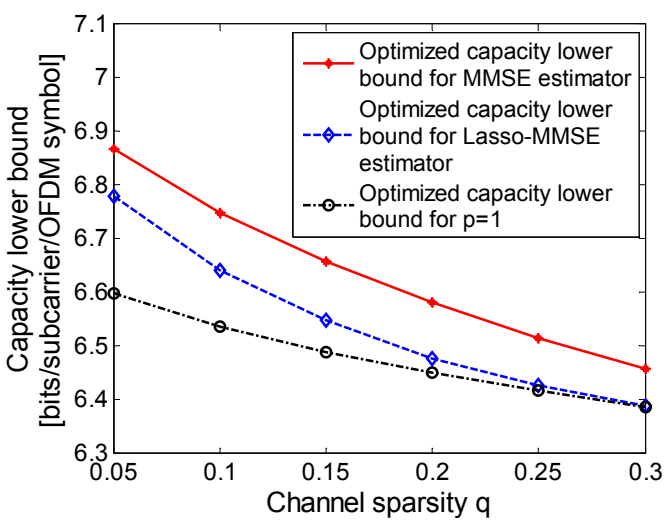

Fig. 3. Optimized capacity lower bound as a function of the sparsity of the channel $q$. 


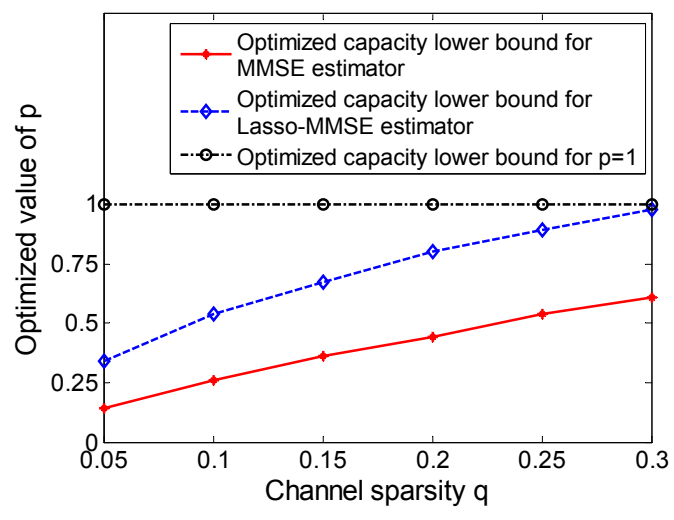

(a) Optimal $p$

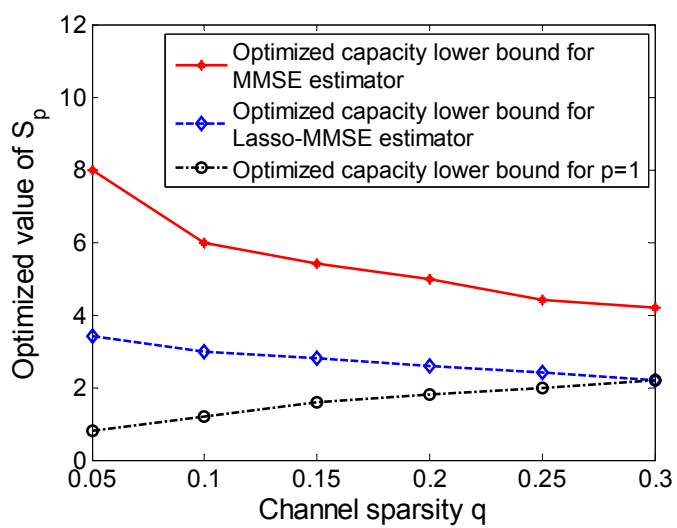

(b) Optimal $S_{p}$

Fig. 4. Optimal $p$ and $S_{p}$ as a function of $q$, when $\gamma=250$.

For the system seting used in Fig. 3, in Fig. 4 we show the values for the optimal $p$ and $S_{p}$.

Figure 4 shows that, for the systems using Lasso-MMSE estimator and MMSE estimator with variable $p$, with the increase of $q$ the optimal values of $p$ increase and the optimal values of $S_{p}$ decrease and for the system with Lasso-MMSE estimator they become very close to those for system using MMSE estimator and $p=1$. Also, for the system that uses Lasso-MMSE the ratio $p / q$ is constantly higher than 3 , and for the system with MMSE it is close to 2. Similar behaviour for the ratio $p / q$ in the optimal solution, was observed in all the experiments. Thus, for the MMSE estimation, $p$ is never too close to $q$ and the iterative solution to (3) is the optimal one [17-19].

To find a polynomial time optimization procedure for solving (6) we investigated the convexity of the optimization problem in different points of the considered region. By numerically evaluating the Hessian matrix of the problem and its eigenvalues, we found that the problem is neither convex nor concave, and thus, the convex optimization approaches are not guaranteed to converge to a global solution. Nevertheless, we found that the for all the tested scenarios for different values of $\gamma_{0}$ and $q$, the capacity lower bound exhibits a behaviour similar to the one shown in Fig. 5.

Figure 5 shows that the capacity lower bound has structure with a single region where its value is very close to the

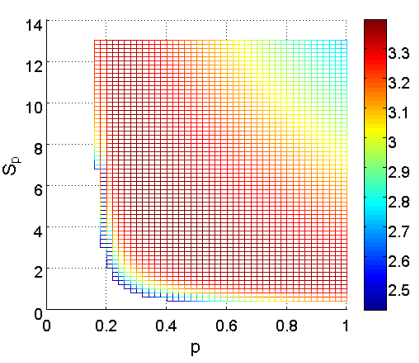

(a) $\underline{C}$ in the range

$\overline{(2.4170,3.3970)}$

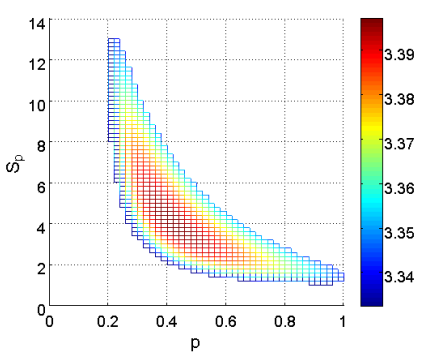

(c) $C$ in the range $(3.3325,3.3970)$

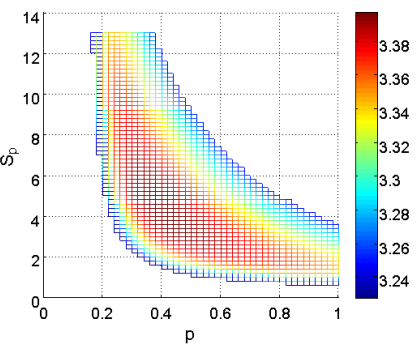

(b) $\underline{C}$ in the range $\overline{(3.2273,3.3970)}$

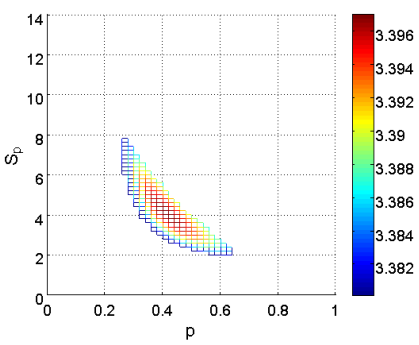

(d) $C$ in the range (3.3800, 3.3970)

Fig. 5. $\underline{C}$ shown in different ranges of its value when $q=0.1$ and $\gamma_{0}=25$.

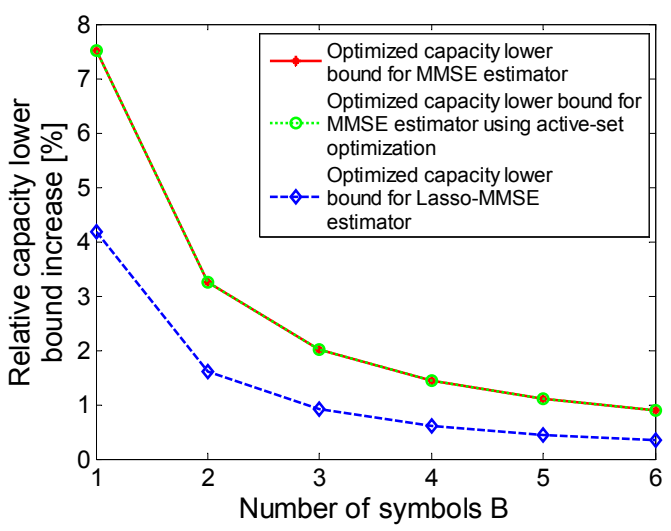

Fig. 6. Relative capacity lower bound increase as a function of the number of OFDM symbols per channel block $B$, when $q=0.1$ and $\gamma_{0}=250$.

optimal for a large area of $p$ and $S_{p}$ values and there are not any local maxima away from this central region. That is why we propose to use any algorithm for nonlinear constrained convex optimization (here we used the fmincon Matlab function with the active-set algorithm with a starting point at $p_{0}=3 q$ and $S_{p 0}=6$ (the starting points do not significantly influence the optimization process as long as $p_{0}>2 q$ and $S_{p 0}$ is not too high)). For very high values of $S_{p}$ and low values of $p$ there is another local maximum for $\underline{C}$ but its value is much lower than the one in the observed region.

To illustrate the effectiveness of the proposed way of estimating the optimal values of $p$ and $S_{p}$ and the optimized 


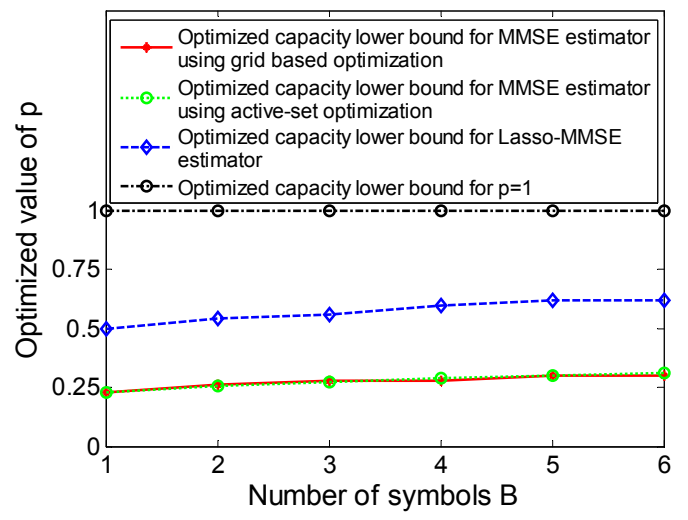

(a) Optimized $p$

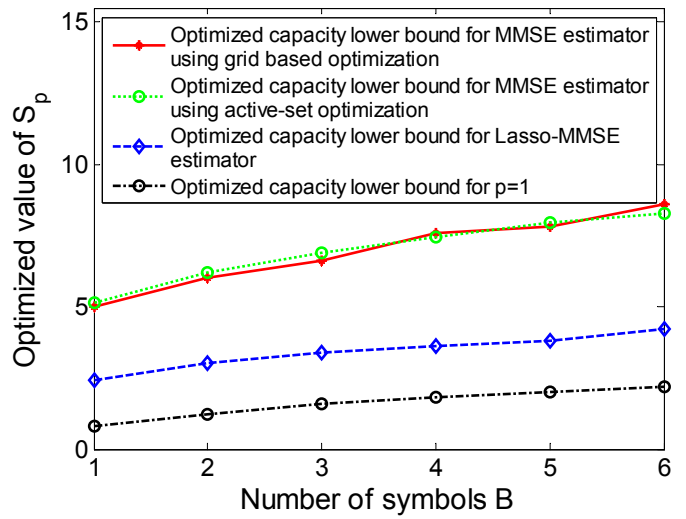

(b) Optimized $S_{p}$

Fig. 7. Optimized values for $p$ and $S_{p}$ when $\gamma_{0}=250$ and $q=0.1$.

capacity lower bound, in Fig. 6 we show the relative increase of the optimized $\underline{C}$ for the three considered estimators, as a function of the number of OFDM symbols per channel block $B$.

Figure 6 shows that the nonlinear constrained convex optimization for (6) provides the same value (to numerical precision due to the grid resolution and the numerical properties of the optimization algorithms) for $\underline{C}$ as the grid based optimization, but uses an algorithm with a polynomial complexity. Additionally, the figure shows that as $B$ increases the relative capacity lower bound increase is reduced, which is due to the larger number of OFDM symbols experiencing the same channel, so low percentage of the overall subcarriers are used as pilot subcarriers and further lowering this number (using improved channel estimation) does not increase the capacity significantly. Also, the MMSE estimator almost doubles the relative capacity lover bound increase compared to the Lasso-MMSE estimator, meaning that there is much space to improve in estimation when using Lasso based CS.

To further observe the performance of the convex optimization applied to (6), in Fig. 7 we show the optimal values for $p$ and $S_{p}$ as a function of $B$.

Figure 7 shows that the optimized values of $p$ and $S_{p}$ obtained by nonlinear convex constrained optimization closely match those obtained by the grid based search. Also, in Fig. 7(b), the convex optimization shows values of $S_{p}$ which are smoother than those of the grid based optimization (obtained as a consequence of the grid with insufficient resolution). This shows that the convex optimization can be efficiently used to solve (6).

\section{Conclusion}

We proposed an improved capacity lower bound of an asymptotic capacity of an OFDM pilot aided communication system with no channel state information at the transmitter on the Bernoulli-Gaussian channel. The improvement is obtained through the substitution of the estimation error of the Lasso-MMSE estimator with the one obtained using the MMSE estimator. Additionally, we analyzed an approach to obtain the optimized capacity lower bound with respect to the fraction of used pilot symbols and the pilot to data power ratio and found that the optimization problem is neither concave nor convex. Nevertheless, the optimization can easily be carried out with a solution sufficiently close to the optimal one, using nonlinear constrained convex optimization.

\section{References}

[1] COSOVIC, I., AUER, G. Capacity of MIMO-OFDM with pilotaided channel estimation. EURASIP Journal on Wireless Commommunications and Networking, 2007, vol. 2007, no. 1, p. 1-12. ISSN: $1687-1499$. DOI: $10.1155 / 2007 / 32460$

[2] VIKALO, H., HASSIBI, B., HOCHWALD, B., et al. On the capacity of frequency- selective channels in training-based transmission schemes. IEEE Transactions on Signal Processing, Sep. 2004, vol. 52, no. 9, p. 2572-2583. ISSN: 1053-587X. DOI: $10.1109 /$ TSP.2004.832020

[3] NAJJAR, L. On optimality limits of channel-structured estimation in multicarrier systems. IEEE Transactions on Vehicular Technology, Jun. 2012, vol. 61, no. 5, p. 2382-2387. ISSN: 0018-9545. DOI: 10.1109/TVT.2012.2192947

[4] HU, D., WANG, X., HE, L. A new sparse channel estimation and tracking method for time-varying OFDM systems. IEEE Transactions on Vehicular Technology, Nov. 2013, vol. 62, no. 9, p. 4648-4653. ISSN: 0018-9545. DOI: 10.1109/TVT.2013.2266282

[5] BERGER, C., ZHOU, S., PREISIG, J., et al. Sparse channel estimation for multicarrier underwater acoustic communication: From subspace methods to compressed sensing. IEEE Transactions on Signal Processing, Mar. 2010, vol. 58, no. 3, p. 1708-1721. ISSN: 1053-587X. DOI: 10.1109/TSP.2009.2038424

[6] TAUBOCK, G., HLAWATSCH, F., EIWEN, D., et al. Compressive estimation of doubly selective channels in multicarrier systems: Leakage effects and sparsity-enhancing processing. IEEE Journal of Selected Topics in Signal Processing, Apr. 2010, vol. 4, no. 2, p. 255-271. ISSN: 1932-4553. DOI: 10.1109/JSTSP.2010.2042410

[7] GUI, G., ADACHI, F. Improved least mean square algorithm with application to adaptive sparse channel estimation. EURASIP Journal on Wireless Communications and Networking, 2013, vol. 2013, no. 1, p. 1-18. ISSN: 1687-1499. DOI: 10.1186/1687-1499-2013-204

[8] MENG, J., YIN, W., LI, Y., et al. Compressive sensing based highresolution channel estimation for OFDM system. IEEE Journal of Selected Topics in Signal Processing, Feb. 2012, vol. 6, no. 1, p. 15-25. ISSN: 1932-4553. DOI: 10.1109/JSTSP.2011.2169649 
[9] BAJWA, W., HAUPT, J., SAYEED, A., et al. Compressed channel sensing: A new approach to estimating sparse multipath channels. Proceedings of the IEEE, Jun. 2010, vol. 98, no. 6, p. 1058-1076. ISSN: 1058-1076. DOI: 10.1109/JPROC.2010.2042415

[10] QI, C., YUE, G., WU, L., et al. Pilot design for sparse channel estimation in OFDM-based cognitive radio systems. IEEE Transactions on Vehicular Technology, Feb. 2014, vol. 63, no. 2, p. 982-987. ISSN: 0018-9545. DOI: 10.1109/TVT.2013.2280655

[11] PEJOSKI, S., KAFEDZISKI, V. Estimation of sparse time dispersive channels in pilot aided OFDM using atomic norm. IEEE Wireless Communications Letters, Aug. 2015, vol. 4, no. 4, p. 397-400. ISSN: 2162-2337. DOI: 10.1109/LWC.2015.2425410

[12] CHENG, P., CHEN, Z., RUI, Y., et al. Channel estimation for OFDM systems over doubly selective channels: A distributed compressive sensing based approach. IEEE Transactions on Communications, Oct. 2013, vol. 61, no. 10, p. 4173-4185. ISSN: 0090-6778. DOI: 10.1109/TCOMM.2013.072813.120758

[13] PEJOSKI, S., KAFEDZISKI, V. Asymptotic capacity lower bound for an OFDM system with Lasso compressed sensing channel estimation for Bernoulli-Gaussian channel. IEEE Communications Letters, Mar. 2015, vol. 19, no. 3, p. 379-382. ISSN: 1089-7798. DOI: 10.1109/LCOMM.2014.2385074

[14] TUlinO, A., CAIRE, G., VERDU, S., et al. Support recovery with sparsely sampled free random matrices. IEEE Transactions on Information Theory, Jul. 2013, vol. 59, no. 7, p. 4243-4271. ISSN: 0018-9448. DOI: 10.1109/TIT.2013.2250578

[15] RANGAN, S., FLETCHER, A., GOYAL, V. Asymptotic analysis of MAP estimation via the replica method and applications to compressed sensing. IEEE Transactions on Information The ory, Mar. 2012, vol. 58, no. 3, p. 1902-1923. ISSN: 0018-9448. DOI: $10.1109 /$ TIT.2011.2177575

[16] HASSIBI, B., HOCHWALD, B. How much training is needed in multiple-antenna wireless links? IEEE Transactions on Information Theory, Apr. 2003, vol. 49, no. 4, p. 951-963. ISSN: 0018-9448. DOI: $10.1109 /$ TIT.2003.809594

[17] MA, J., YUAN, X., PING, L. Turbo compressed sensing with partial DFT sensing matrix. IEEE Signal Processing Letters, Feb. 2015, vol. 22, no. 2, p. 158-161. ISSN: 1070-9908. DOI: 10.1109/LSP.2014.2351822
[18] MA, J., YUAN, X., PING, L. On the performance of turbo signal recovery with partial DFT sensing matrices. IEEE Signal Processing Letters, Oct. 2015, vol. 22, no. 10, p. 1580-1584. ISSN: 1070-9908. DOI: 10.1109/LSP.2015.2414951

[19] WEN, C. K., WONG, K. K. Analysis of compressed sensing with spatially-coupled orthogonal matrices. CoRR Journal, 2014, vol. 1402.3215, no. 1, p. 1-5. [Online]. Cited 2015-12-31. Available at: http://arxiv.org/abs/1402.3215

\section{About the Authors ...}

Slavche PEJOSKI was born in 1984. He received his $\mathrm{PhD}$ degree from the Faculty of Electrical Engineering and Information Technologies at the University "Ss. Cyril and Methodius" in Skopje, Republic of Macedonia in 2015. His research interests include compressed sensing, channel estimation, wireless communications and video.

Venceslav KAFEDZISKI was born in 1958. He earned his BS and MSc degree from the Faculty of Electrical Engineering (now Faculty of Electrical Engineering and Information Technologies) at the University "Ss. Cyril and Methodius" in Skopje, Republic of Macedonia in 1982 and 1989 respectively, and his Ph.D. degree from Arizona State University, Tempe, USA, in 2000. In 2001 he joined the Faculty of electrical engineering in Skopje as an assistant professor, and currently holds a position of a full professor. Dr. Kafedziski is the author of around 60 scientific publications published in prestigious international journals and conference proceedings in the areas of wireless communications, information theory and signal processing. Since 2008 he has been the chair of the Technical Committee for Electronic Communications at the Institute for Standardization of Republic of Macedonia. He is the organizer of the IEEE Republic of Macedonia Section Information Theory Society Chapter. 